\title{
Adenoviral Transduced hIL-12-expressing Autologous Dendritic Cells INXN-3001 Plus Activator Ligand INXN-1001
}

National Cancer Institute

\section{Source}

National Cancer Institute. Adenoviral Transduced h/L-12-expressing Autologous

Dendritic Cells INXN-3001 Plus Activator Ligand INXN-1001. NCI Thesaurus. Code

C101789.

Autologous dendritic cells tranduced with a replication incompetent adenovirus encoding human pro-inflammatory cytokine interleukin-12 (IL-12) (INXN-3001) in combination with the proprietary orally bioavailable, small molecule activator ligand INXN-1001, with potential immunomodulating and antineoplastic activities. Production of IL-12 is controlled by an inducible DNA element that allows transcription initiation only in the presence of the ligand inducer INXN-1001. Upon intratumoral injection of INXN-3001 and subsequent oral administration of activator ligand, INXN-1001 is able to induce expression of IL-12 in INXN-3001. IL-12 expressed by the adenovirus may activate the immune system by promoting the activation of natural killer cells, inducing the secretion of interferon-gamma and inducing a cytotoxic T lymphocyte response against tumor cells, which may result in immune-mediated tumor cell death and inhibition of tumor cell proliferation. As INXN-1001 regulates both the timing and the levels of IL-12 expression, IL-12 toxicity can be reduced. 